\title{
ENSINO POR COMPETÊNCIAS NAS DISCIPLINAS DE CONSTRUÇÃO CIVIL - CASO DA UTFPR GUARAPUAVA
}

Marcela Maier Farias Czap-mfarias@utfpr.edu.br

UTFPR Guarapuava, COECI

Av. Prof. Laura Pacheco Bastos, 800.

CEP 85053-525 - Guarapuava - Paraná

Viviane Chulek-vivianechulek@utfpr.edu.br

UTFPR Guarapuava, DEPED

Av. Prof. Laura Pacheco Bastos, 800.

CEP 85053-525 - Guarapuava - Paraná

Marly Terezinha Quadri Simões da Silva-marlytsilva@utfpr.edu.br

UTFPR Guarapuava, COECI

Av. Prof. Laura Pacheco Bastos, 800.

CEP 85053-525 - Guarapuava - Paraná

Rodrigo Scoczynski Ribeiro - rodrigosribeiro@utfpr.edu.br

UTFPR Guarapuava, COECI

Av. Prof. Laura Pacheco Bastos, 800.

CEP 85053-525 - Guarapuava - Paraná

Dyorgge Alves Silva-dyorggeasilva@utfpr.edu.br

UTFPR Guarapuava, COECI

Av. Prof. Laura Pacheco Bastos, 800.

CEP 85053-525 - Guarapuava - Paraná

Resumo: As mudanças exigidas dos cursos de graduação de Engenharia são motivadas pelo avanço científico e tecnológico e nas transformações das relações e necessidades da sociedade. A publicação das novas Diretrizes Curriculares Nacionais do Curso de Graduação em Engenharia buscou sintetizar as necessidades de mudança e impulsionou o processo de inovação curricular nos cursos de engenharia do país, assim como na Universidade Tecnológica Federal do Paraná - UTFPR. A concepção sobre a formação de engenheiros precisa alcançar competências profissionais e significa mobilizar e integrar conhecimentos em situações de uso realistas, com contexto social, cultural, econômico e científico. Foi necessário renovar as perspectivas para transformar a proposta curricular com abordagem por competências que incluam além do conhecimento (saber), também o saber-fazer e o saber-ser. Este trabalho apresenta as estratégias que foram adotadas na reformulação do currículo no que se refere às disciplinas da área de Construção Civil do Curso de Engenharia Civil da UTFPR, campus Guarapuava. Além de expor a metodologia de trabalho aplicada pelo Núcleo Docente Estruturante do curso, são apresentadas como exemplo três disciplinas da área, onde os temas de estudos e resultados de aprendizagem têm características peculiares ou inovadoras.

Palavras-chave: Diretrizes Curriculares. Currículo por competências. Construção Civil. 


\section{INTRODUÇÃO}

A publicação das novas Diretrizes Curriculares Nacionais do Curso de Graduação em Engenharia (BRASIL, 2019), que tem como objetivos elevar a qualidade do ensino em Engenharia no país, permitir maior flexibilidade na estruturação dos cursos de forma a permitir a inovação curricular, reduzir a taxa de evasão e ainda direcionar para o atendimento de demandas futuras quando a formação de engenheiros, impõe a necessidade não apenas de revisão curricular, mas de mudança de concepção sobre a formação de engenheiros na UTFPR. Assim, essa concepção passa a assumir o compromisso com o desenvolvimento de competências profissionais que pressupõe muito mais que a apropriação do conhecimento científico, mas a necessidade de mobilizar e integrar conhecimentos em situações de uso realistas, com contexto social, cultural, econômico e científico. Por isso, o objetivo deste trabalho é apresentar as estratégias que foram adotadas na reformulação do currículo no que se refere as disciplinas da área de Construção Civil do Curso de Engenharia Civil da UTFPR, campus Guarapuava.

\section{O CURRÍCULO POR COMPETÊNCIAS}

A mudança curricular exigida pela nova DCN pode ser descrita como uma transformação de um currículo disciplinar em um currículo integrado. LOPES (2008) descreve o currículo disciplinar como consequência de princípios de organização curricular baseados na lógica das ciências ou na natureza do conhecimento, enquanto que currículos integrados são entendidos como algo baseado nos interesses e necessidades dos alunos e na relevância social do conhecimento.

Tendo em vista que o propósito da educação superior, alicerçado na relação intrínseca entre trabalho e educação, a construção do currículo por competências, apresenta-se como uma possibilidade real para o desenvolvimento de um programa formativo sistemático que proporciona o desenvolvimento do estudante.

A análise cuidadosa acerca do conceito de competência permite observar que esse conceito agrega três tipos de saberes: o saber, o saber-fazer e o saber ser. O saber relaciona-se aos conhecimentos que já fazem parte dos currículos acadêmicos e não devem ser minimizados ou negligenciados em um currículo por competências. O saber-fazer pressupõe um saber agir pautado na mobilização, integração e transferência de conhecimentos para diferentes situações. O saber ser relaciona ao propósito de formação humanística podendo ser solicitados previamente para uma aprendizagem, desenvolvidos como objetivo de formação ainda como critérios de qualidade particulares a certos desempenhos (SCALLON, 2015).

\section{METODOLOGIA}

Os trabalhos iniciaram com a participação de membros do Núcleo Docente Estruturante (NDE) e da pedagoga do campus em uma Oficina de Design de Cursos, onde, em linhas gerais, refletiu-se sobre o curso de Engenharia Civil no momento atual, culminando na construção do perfil do egresso, das competências a serem desenvolvidas e dos elementos que as constituem para todos os cursos de Engenharia Civil da UTFPR.

O perfil do egresso foi definido como: "profissionais que se caracterizam por criar soluções de engenharia para o desenvolvimento sustentável do ambiente construído, levando em consideração as demandas da sociedade. Poderão atuar na indústria da construção civil e em órgãos governamentais a ela associados. Para tanto, são capazes de projetar sistemas, produtos e processos relacionados à construção civil considerando a ética, a segurança, a legislação e os impactos ambientais; implementar obras de construção civil de forma responsável, eficaz e com 
liderança de equipes multiprofissionais; gerenciar organizações correlatas à indústria da construção civil e/ou recursos naturais, de forma empreendedora, inovadora e sustentável".

Sendo que, para alcançar este perfil, o estudante deve desenvolver 6 competências, são elas:

- INVESTIGAR: problemas de contexto real integrando conhecimentos técnicocientíficos da literatura, selecionando informações relevantes e aplicando em soluções mais adequadas às contingências com integridade autonomia reflexiva e com senso crítico;

- RESOLVER: problemas estruturados de diferentes contextos da engenharia, de maneira autônoma, integrando os fundamentos de química, física e matemática, o raciocínio lógico e as ferramentas tecnológicas;

- INTERVIR: em situações conflituosas, fundamentado em conhecimentos da dimensão social, econômica, cultural, tecnológica e ambiental, demonstrando autonomia, comunicação qualificada, responsabilidade, autoria e autenticidade;

- EXECUTAR: projetos de construção civil, integrando a interpretação do projeto, o plano operacional e de viabilidade, a execução, o monitoramento retroalimentado e o replanejamento, com prioridade na utilização racional de recursos materiais, financeiros e tecnológicos, e na coordenação regulada de equipes multiprofissionais, de modo responsável, eficiente e cooperativo;

- PROJETAR: sistemas em diferentes contextos da construção civil, articulando adequadamente análise de demanda, diagnóstico de viabilidade, busca de referências, seleção de concepções e emprego de ferramentas tecnológicas, conforme os aspectos de funcionalidade, segurança e sustentabilidade, de forma crítico-reflexiva, criativa e com responsabilidade social;

- GERENCIAR: diferentes organizações correlatas à indústria da construção civil, com visão sistêmica, empregando planejamento, metodologias e ferramentas gerenciais e tecnológicas devidamente validados para tomadas de decisão sustentáveis e inovadoras, com responsabilidade socioambiental, de forma empreendedora, colaborativa e reflexiva.

Posteriormente, em encontros do NDE, foram elencados elementos, a fim de criar etapas de desenvolvimento nas competências, chamados de elementos de competência (EC). Cada disciplina pode se responsabilizar totalmente ou em partes pelo desenvolvimento dos ECs.

Os professores das diferentes áreas que compõem o curso reuniram-se para transformar as ementas das disciplinas em temas de estudo (TE), descrevendo melhor os conteúdos a serem trabalhados. A partir dos TEs e ECs, aos quais a disciplina contribuí, os resultados de aprendizagem (RAs) foram construídos. O aprimoramento dos TEs e RAs foi realizado de forma coletiva em encontros do NDE, com a participação de todos os professores das áreas específicas do curso e do Departamento de Educação.

\section{RESULTADOS}

A consolidação da área de Construção Civil no curso se expressa na oferta das disciplinas apresentadas no Quadro 1.

Quadro 1 - Disciplinas da área de Construção Civil do curso de Engenharia Civil da UTFPR, Guarapuava.

\begin{tabular}{|c|c|c|c|}
\hline Código & Disciplina & $\mathrm{CH}(\mathrm{hrs})$ & Período \\
\hline INT & Introdução à Engenharia & 60 & $1^{\circ}$ \\
\hline DPA & Desenho de Projeto Arquitetônico & 75 & $1^{\circ}$ \\
\hline MCC & Materiais de Construção Civil & 60 & $2^{\circ}$ \\
\hline MIC & Modelagem da Informação da Construção & 75 & $2^{\circ}$ \\
\hline PTC & Processos e Técnicas da Construção Civil & 60 & $3^{\circ}$ \\
\hline SCP & Sistemas Complementares e Planejamento na Construção Civil & 60 & $4^{\circ}$ \\
\hline IEL & Instalações Elétricas & 75 & $6^{\circ}$ \\
\hline AEC & Argamassas e Concretos & 60 & $6^{\circ}$ \\
\hline
\end{tabular}


"Os desafios para formar hoje o engenheiro do amanhã"

\section{Evento On-line}

\begin{tabular}{|c|c|c|c|}
\hline IHI & Instalações Hidrossanitárias & 75 & $7^{\circ}$ \\
\hline QDE & Qualidade e Desempenho das Edificações & 60 & $8^{\circ}$ \\
\hline
\end{tabular}

Fonte: os autores.

A aprendizagem de cada EC é evolutiva e, geralmente, se dá através de um grupo de RAs que, individualmente, aparecem em mais de uma disciplina. Nesse sentido, os professores dividiram cada EC em momentos internalizadores (destacados em amarelo na Figura 1) e mobilizadores (em laranja). O caráter internalizador é o que se refere a conhecimentos inéditos aos estudantes e o mobilizador, diz respeito aos conteúdos que mobilizam conhecimentos que o estudante já teve contato, e que serão desenvolvidos na disciplina. Em lilás estão destacados RAs das disciplinas do nono período, que irão acontecer em paralelo com a disciplina certificadora da competência GERENCIAR. Sendo assim, a evolução de complexidade dos ECs e das características internalizadoras e mobilizadoras podem ser constatados na diagonalização da matriz, mostrada na Figura 1, onde as colunas representam os ECs e as linhas as disciplinas de todo o curso.

Figura 1 - Matriz completa do curso de Engenharia Civil da UTFPR, Guarapuava.

\begin{tabular}{|c|c|c|c|c|c|c|c|}
\hline & Competência & INVESTIGAR & RESOLVER & INTERVIR & EXECUTAR & PROJETAR & GERENCIAR \\
\hline & Disciplina/EC & \begin{tabular}{|l|l|l|l|l|l|l|l|l|}
$D$ & P & F & D & V & $A$ & $A$ & $D$ \\
\end{tabular} & \begin{tabular}{|l|l|l|l|l|l|} 
I & D & E & D & $V$ & $R$ \\
\end{tabular} & \begin{tabular}{|l|l|l|l|l|l|} 
R & F & S & $A$ & $D$ & I \\
\end{tabular} & \begin{tabular}{|l|l|l|l|l|l|} 
I & P & $S$ & $G$ & $M$ & $D$ \\
\end{tabular} & \begin{tabular}{|l|l|l|l|l|l|}
$A$ & I & $A$ & $D$ & $R$ & $V$ \\
\end{tabular} & \begin{tabular}{|l|l|l|l|l|l|}
$A$ & $F$ & $E$ & I & $M$ & $D$ \\
\end{tabular} \\
\hline & 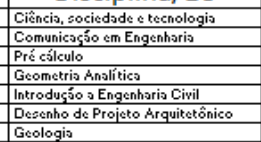 & $\begin{array}{|ccc|} & & 1 \\
1 & 1 & 1 \\
1 & 1 & 1 \\
\end{array}$ & $\begin{array}{ccc}1 & & 1 \\
1 & \vdots \\
1 & & 1 \\
& & 1\end{array}$ & 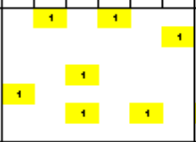 & 1 & & \\
\hline & 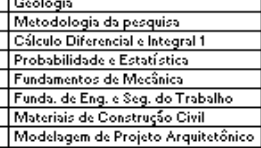 & 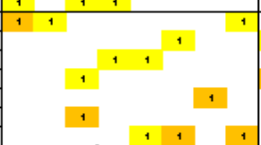 & 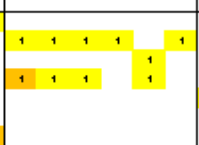 & +1 & $: 1^{1}$ & & \\
\hline & 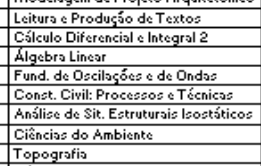 & 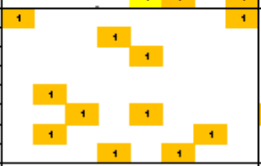 & $\left|\begin{array}{cccc}1 & 1 & 1 & \\
& & \vdots & \\
1 & & & 1\end{array}\right|$ & $\begin{array}{|lll|} & & \\
& & \\
& & \\
+ & 1 & 1 \\
\end{array}$ & 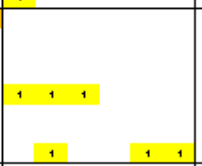 & & \\
\hline 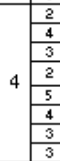 & 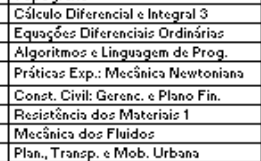 & & 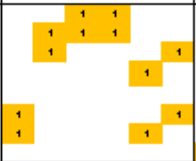 & 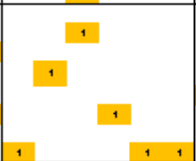 & $\begin{array}{lllll}1 & 1 & 1 & 1 & 1\end{array}$ & $\cdot$ & \\
\hline 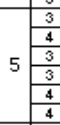 & 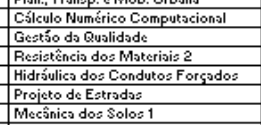 & & & $\begin{array}{|llll|} & 1 & & \\
& & 1 & \\
1 & & & 1 \\
\end{array}$ & 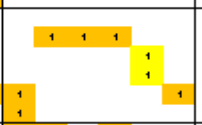 & $\begin{array}{ll}1 & 1 \\
1 & 1 \\
& 1 \\
1 & 1 \\
\end{array}$ & \\
\hline 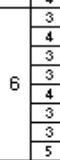 & 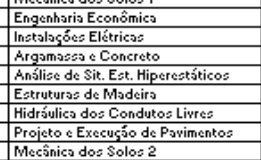 & & & & 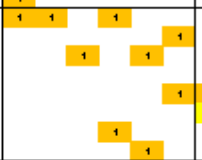 & 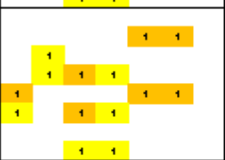 & \\
\hline 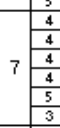 & 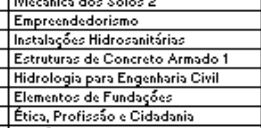 & & & & & 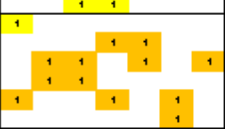 & $\mid \begin{array}{lll}1 & 1 & 1 \\
1 & 1 & \\
1 & 1 & 1 \\
1 & 1 & 1\end{array}$ \\
\hline \begin{tabular}{l|l}
3 \\
3 \\
\cline { 1 - 2 } 2 \\
\cline { 2 - 2 } 3 \\
\cline { 2 - 2 } 3 \\
\cline { 2 - 2 } 3 \\
3
\end{tabular} & 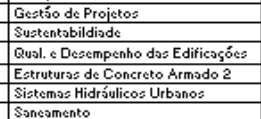 & & & & & & \begin{tabular}{|lllll}
1 & 1 & 1 & 1 & \\
1 & 1 & 1 & & 1 \\
& 1 & & & $\vdots$ \\
1 & & & 1 & 1
\end{tabular} \\
\hline & 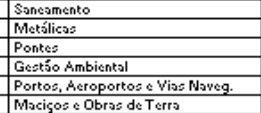 & & & & & & $\begin{aligned} \cdot \\
. \\
\end{aligned}$ \\
\hline
\end{tabular}

Fonte: os autores.

Em reunião com o NDE do curso, as disciplinas de Construção Civil foram relacionadas com os ECs mais adequados para a área, como mostra o Quadro 2. 
Quadro 2 - Elementos de Competência das disciplinas da área de Construção Civil do curso de Engenharia Civil da UTFPR, Guarapuava.

\begin{tabular}{|c|c|c|c|c|c|c|c|}
\hline$(\mathrm{C})$ & Elemento de Competência (EC) & & 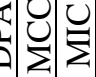 & $\mid \begin{array}{l}0 \\
\qquad\end{array}$ & 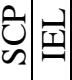 & $\mid$ & 国嘼 \\
\hline \multirow{7}{*}{ 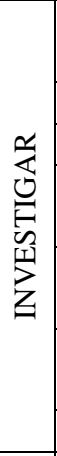 } & $\begin{array}{c}\text { Descrever: situações envolvendo problemas de contexto real e fundamentado na } \\
\text { literatura tecno-científica e em fontes fidedignas. }\end{array}$ & & & & & & \\
\hline & Problematizar: situações de contexto real descritas. & & & & & & \\
\hline & Formular: hipóteses a partir de problemas selecionados. & & & & & & \\
\hline & $\begin{array}{l}\text { Validar: hipóteses aplicando métodos, estratégias ou procedimentos definidos, } \\
\text { com integridade e autonomia. }\end{array}$ & & & & & & \\
\hline & $\begin{array}{c}\text { Analisar: resultados de validação, replanejando método, estratégia ou } \\
\text { procedimento de forma reflexiva. }\end{array}$ & & & & & & \\
\hline & $\begin{array}{l}\text { Argumentar: de forma consistente e crítica e pautado em conhecimentos } \\
\text { técnico-científicos da literatura, em defesa de resultados analisados. }\end{array}$ & & & & & & \\
\hline & Documentar: o processo de pesquisa. & & & & & & \\
\hline \multirow{2}{*}{$\begin{array}{l}\text { ב̇ } \\
\text { on } \\
\text { TI } \\
\simeq\end{array}$} & Interpretar: os problemas estruturados no contexto das Engenharias. & & & & & & \\
\hline & $\begin{array}{l}\text { Determinar: a solução por meio de linguagem matemática e raciocínio lógico, } \\
\text { empregando ferramentas tecnológicas adequadas à hipótese. }\end{array}$ & & & & & & \\
\hline \multirow{3}{*}{ 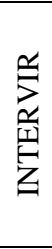 } & $\begin{array}{c}\text { Reconhecer: situações de conflito de ordem individual e coletiva delimitando } \\
\text { variáveis em situações de contexto real. }\end{array}$ & & & & & & \\
\hline & $\begin{array}{l}\text { Selecionar: ferramentas, modelos e conceitos que possibilitem ampliar a } \\
\text { compreensão da situação identificada. }\end{array}$ & & & & & & \\
\hline & $\begin{array}{l}\begin{array}{l}\text { Definir: estratégias de intervenção autênticas e autorais adequadas à resolução } \\
\text { do conflito tratado. }\end{array} \\
\end{array}$ & & & & & & \\
\hline \multirow{6}{*}{ 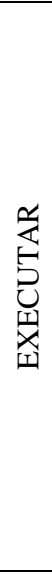 } & $\begin{array}{l}\text { Interpretar: soluções propostas para a construção civil fundamentado em } \\
\text { técnicas, normas e conceitos da área. }\end{array}$ & & & & & & \\
\hline & $\begin{array}{c}\text { Planejar: a operacionalização de técnicas, recursos físicos e financeiros e de } \\
\text { organização de equipes de trabalho conforme propostas de soluções } \\
\text { interpretadas. }\end{array}$ & & & & & & \\
\hline & $\begin{array}{l}\text { Selecionar: critérios de Segurança e Saúde do Trabalho, controle de qualidade e } \\
\text { sustentabilidade, conforme o planejamento de execução do empreendimento. }\end{array}$ & & & & & & \\
\hline & $\begin{array}{l}\text { Gerenciar: equipes multiprofissionais, recursos materiais, tecnológicos e } \\
\text { financeiros, conforme o planejamento de execução do empreendimento e os } \\
\text { critérios selecionados, de modo eficiente e cooperativo. }\end{array}$ & & & & & & \\
\hline & $\begin{array}{l}\text { Monitorar: a execução e os resultados alcançados, com base nos critérios } \\
\text { selecionados, replanejando, se necessário. }\end{array}$ & & & & & & \\
\hline & $\begin{array}{l}\text { Documentar: o processo de gerenciamento do empreendimento, de forma } \\
\text { responsável. }\end{array}$ & & & & & & \\
\hline \multirow{4}{*}{ 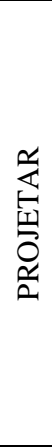 } & $\begin{array}{c}\text { Identificar: os parâmetros técnicos, legais e socioeconômicos necessários para a } \\
\text { elaboração do projeto, seus prazos bem como as ferramentas tecnológicas } \\
\text { aplicáveis, com responsabilidade social durante todas as etapas. }\end{array}$ & & & & & & \\
\hline & $\begin{array}{c}\text { Dimensionar: os elementos dos projetos com ferramentas tecnológicas } \\
\text { adequadas, considerando critérios de segurança e sustentabilidade, com atitudes } \\
\text { criativa e crítico-reflexiva. }\end{array}$ & & & & & & \\
\hline & $\begin{array}{c}\text { Representar: graficamente, as informações necessárias à implementação dos } \\
\text { elementos das edificações, empregando ferramentas tecnológicas selecionadas e } \\
\text { conforme o dimensionamento. }\end{array}$ & & & & & & \\
\hline & $\begin{array}{l}\text { Validar: os próprios projetos e os de pares de acordo com os critérios vigentes } \\
\text { nas referências empregadas. }\end{array}$ & & & & & & \\
\hline \multirow{3}{*}{ 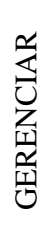 } & $\begin{array}{l}\text { Elaborar: Plano de Ação para as soluções formuladas, considerando métodos, } \\
\text { ferramentas gerenciais e indicadores de desempenho, correlatos à indústria da } \\
\text { construção civil. }\end{array}$ & & & & & & \\
\hline & $\begin{array}{l}\text { Monitorar: continuamente os indicadores de desempenho, conforme os critérios } \\
\text { do Plano de Ação, reformulando-o com base nos resultados, de forma reflexiva. }\end{array}$ & & & & & & \\
\hline & Documentar: o processo de gerenciamento da organização, de forma reflexiva. & & & & & & \\
\hline
\end{tabular}

Fonte: os autores. 
Dentre as disciplinas da área de Construção Civil, três foram escolhidas para exemplificar a metodologia desenvolvida. São elas:

- Materiais da Construção Civil: devido a sua inter-relação entre TEs e RAs não usual;

- Modelagem da Informação da Construção Civil: pelo caráter inovador, relacionado a um tema que ainda não é amplamente abordado nas universidades públicas;

- Qualidade e Desempenho das Edificações: por abordar a norma de desempenho de edificações que estabelece parâmetros mínimos a serem obedecidos na construção civil.

Para a disciplina de Materiais de Construção Civil, apresentada no Quadro 3, foram desenvolvidos 9 TEs e 4 RAs que se relacionam entre si de maneira a abordar os materiais empregados na construção civil de forma sequencial.

Embora a carga horária da disciplina seja de 60 horas e o convencional, no currículo por competências, seja de que um TE seja o equivalente aos conhecimentos desenvolvidos em 10 horas de trabalho, optou-se pelo arranjo dos TEs de acordo com os grupos de materiais típicos da construção civil que devem ser abordados nessa disciplina. O único TE de conotação geral é o TE 1 que aborda a introdução ao formato como a ciência dos materiais desenvolve seus estudos.

O desenvolvimento de RAs que se relacionassem com todos os TEs específicos se deu devido ao fato de que a disciplina tem em sua identidade $50 \%$ de aulas práticas em laboratório. Portanto, embora os ensaios específicos se diferenciem de acordo com os materiais estudados, o resultado que se espera do aprendizado do estudante é semelhante para todos os materiais: (a) que ele os conheça (RA 1); (b) que saiba desenvolver ensaio específico de forma adequada (RA 2); (c) que saiba interpretar as observações laboratoriais (RA 3) e (d) que saiba sugerir formas de utilização desses materiais assertivamente (RA 4).

Quadro 3 - TE e RA da disciplina de Materiais de Construção Civil.

\begin{tabular}{|c|c|c|c|}
\hline \multicolumn{4}{|c|}{ Disciplina de Materiais de Construção Civil (Carga Horária total de 60 horas) } \\
\hline \multicolumn{3}{|r|}{ Temas de Estudo: } & $\mathrm{CH}(\mathrm{h})$ \\
\hline \multicolumn{2}{|c|}{ TE 1} & Propriedades dos materiais: características e suas grandezas. & 5 \\
\hline TE 2 & & Materiais metálicos: metais e ligas, aço, ensaios físicos. & 10 \\
\hline TE 3 & \multicolumn{2}{|r|}{ Madeiras: origem, classificação, beneficiamento, propriedades e ensaios físicos. } & 10 \\
\hline TE 4 & \multicolumn{2}{|r|}{ Materiais argilosos: composição e processos de fabricação, aplicações na const. civil. } & 5 \\
\hline TE 5 & & 5 \\
\hline TE 6 & \multicolumn{2}{|r|}{ Materiais plásticos: polímeros, processamento dos polímeros, aplicações na const. civil. } & 5 \\
\hline TE 7 & \multicolumn{2}{|r|}{ Materiais cerâmicos: produção e aplicações na construção civil. } & 10 \\
\hline TE 8 & \multicolumn{2}{|r|}{ Materiais betuminosos: produção e aplicações na construção civil. } & 5 \\
\hline TE 9 & \multicolumn{2}{|r|}{ Tintas e vernizes: composição, tipos e aplicações na construção civil. } & 5 \\
\hline \multicolumn{4}{|c|}{ Resultados de Aprendizagem: } \\
\hline $\mathrm{C}$ & ECs & \multicolumn{2}{|l|}{ RAs } \\
\hline$\stackrel{\overrightarrow{0}}{\grave{D}}$ & Formular & \multicolumn{2}{|c|}{$\begin{array}{l}\text { RA } 1 \text { (relacionado aos TEs } 1 \text { a 9): Identificar a influência das grandezas e das } \\
\text { propriedades dos materiais considerando as áreas de aplicação da ciência dos materiais } \\
\text { no contexto da engenharia civil de forma reflexiva. }\end{array}$} \\
\hline 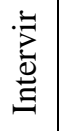 & Definir & \multicolumn{2}{|c|}{$\begin{array}{c}\text { RA } 4 \text { (relacionados aos TEs } 1 \text { a 9): Selecionar os materiais adequadamente para a } \\
\text { utilização na construção civil diferenciando as propriedades e grandezas e o contexto de } \\
\text { aplicação de forma crítica. }\end{array}$} \\
\hline \multirow{2}{*}{ 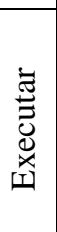 } & Interpretar & \multicolumn{2}{|l|}{$\begin{array}{l}\text { RA } 3 \text { (relacionado aos TEs } 2 \text { a 9): Interpretar os resultados dos ensaios laboratoriais } \\
\text { considerando as normas técnicas pertinentes com responsabilidade. }\end{array}$} \\
\hline & Planejar & \multicolumn{2}{|c|}{$\begin{array}{l}\text { RA } 2 \text { (relacionado aos TEs } 2 \text { a 9): Realizar ensaio laboratorial normativo escolhendo os } \\
\text { procedimentos, equipamentos e insumos adequados à caracterização do material em } \\
\text { estudo de forma colaborativa. }\end{array}$} \\
\hline
\end{tabular}

Fonte: os autores. 
Na disciplina de Modelagem da Informação da Construção Civil, apresentada no Quadro 4, a inovação curricular fica exemplificada através da abordagem de um tema atual, envolvendo novo conceito de visualização 3D com nova ferramenta de modelagem virtual, que tradicionalmente, não é desenvolvido nas disciplinas dos cursos de Engenharia Civil nas universidades públicas. Um dos motivos pode ser a falta de qualificação do corpo docente nos softwares utilizados nessa abordagem, denominada BIM - Building Information Modeling. Porém, este grupo de professores entende que a qualificação continuada é necessária, uma vez que o egresso deste curso concorre por vagas de trabalho juntamente com egressos de IES particulares, onde é comum verificar esse tipo de conhecimento na grade curricular.

A disciplina ainda traz uma abordagem mais complexa no que diz respeito aos projetos arquitetônicos, proporcionando a continuidade do conhecimento iniciado na disciplina de Desenho de Projeto Arquitetônico. No primeiro momento, o estudante desenvolveu um projeto arquitetônico residencial unifamiliar, e aqui, ele desenvolve um projeto arquitetônico residencial multifamiliar e entra em contato com desafios e legislações mais abrangentes, tendo que adequar o projeto através de uma gama maior de exigências.

Quadro 4 - TE e RA da disciplina de Modelagem da Informação da Construção Civil.

Disciplina de Modelagem da Informação da Construção Civil (Carga Horária total de 75 horas)

\begin{tabular}{|c|c|c|c|}
\hline \multicolumn{4}{|c|}{ Modelagem da Informaç } \\
\hline \multicolumn{3}{|r|}{ Temas de Estudo: } & $\mathrm{CH}(\mathrm{h})$ \\
\hline TE 1 & \multicolumn{2}{|r|}{$\begin{array}{c}\text { Modelagem da Informação da Construção (BIM - 3D): visão geral, ferramentas e } \\
\text { aplicação via software. }\end{array}$} & 5 \\
\hline TE 2 & \multicolumn{2}{|r|}{ Código de posturas e obras aplicado a residências multifamiliares verticais. } & 5 \\
\hline TE 3 & \multicolumn{2}{|r|}{ Aspectos de segurança contra incêndios em projetos multifamiliares verticais. } & 5 \\
\hline TE 4 & \multicolumn{2}{|c|}{$\begin{array}{c}\text { Representação de projeto: Topografia, paredes, portas, janelas, forro, mobiliário, tabelas, } \\
\text { quantitativos e impressão. }\end{array}$} & 10 \\
\hline TE 5 & \multicolumn{2}{|r|}{ A multirrepresentação de projetos de edifícios: projetos complementares. } & 10 \\
\hline TE 6 & \multicolumn{2}{|r|}{ Detalhes construtivos de edifícios: escadas, elevadores e desenho universal. } & 10 \\
\hline TE 7 & & Projeto arquitetônico habitacional multifamiliar vertical. & 30 \\
\hline \multicolumn{4}{|c|}{ Resultados de Aprendizagem: } \\
\hline $\mathrm{C}$ & ECs & \multicolumn{2}{|l|}{ RAs } \\
\hline \multirow{3}{*}{ 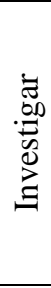 } & Validar & \multicolumn{2}{|c|}{$\begin{array}{l}\text { RA } 5 \text { (relacionado ao TE 6): Analisar os resultados de modelagem em detalhes } \\
\text { construtivos de projetos de edifícios multipavimantos de maneira assertiva e autônoma. }\end{array}$} \\
\hline & Analisar & \multicolumn{2}{|c|}{$\begin{array}{l}\text { RA } 4 \text { (relacionado ao TE 5): Analisar a compatibilidade entre projetos complementares, } \\
\text { considerando uma situação proposta, de maneira assertiva. }\end{array}$} \\
\hline & Documentar & \multicolumn{2}{|c|}{$\begin{array}{l}\text { RA } 6 \text { (relacionado ao TE 7): Elaborar a documentação gráfica a partir da modelagem de } \\
\text { projetos habitacionais multifamiliares verticais, considerando demandas propostas, de } \\
\text { maneira autoral e colaborativa. }\end{array}$} \\
\hline \multirow{2}{*}{$\stackrel{\sum_{0}^{ \pm}}{=}$} & Selecionar & \multicolumn{2}{|c|}{$\begin{array}{l}\text { RA } 1 \text { (relacionado ao TE 1): Reconhecer a aplicabilidade de uma ferramenta } \\
\text { computacional BIM, considerando o desenvolvimento de projetos arquitetônicos, de } \\
\text { maneira crítica e reflexiva. }\end{array}$} \\
\hline & Definir & \multicolumn{2}{|c|}{$\begin{array}{l}\text { RA } 2 \text { (relacionado aos TEs } 2 \text { e } 3 \text { ): Identificar restrições nos projetos multifamiliares } \\
\text { verticais considerando regulamentos, código de posturas e obras municipal e aspectos de } \\
\text { segurança contra incêndios, de maneira autônoma e crítica. }\end{array}$} \\
\hline 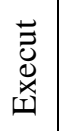 & Interpretar & \multicolumn{2}{|l|}{$\begin{array}{l}\text { RA } 3 \text { (relacionado ao TE 4): Modelar adequadamente um projeto arquitetônico } \\
\text { multifamiliar vertical, considerando todos os elementos componentes e as normas } \\
\text { técnicas, de maneira autorregulada e crítica. }\end{array}$} \\
\hline
\end{tabular}

Fonte: os autores.

Qualidade e Desempenho das Edificações, apresentada no Quadro 5, é uma disciplina que foi criada com o objetivo de atender à demanda do atual cenário consumidor, efetivado na NBR 15.575 que aborda a eficiência e o bom desempenho das edificações habitacionais. Esta norma atinge as profissões relacionadas com a Construção Civil, desde fornecedores dos materiais, projetistas e construtores, e tem como objetivo garantir conforto, acessibilidade, higiene, estabilidade e vida útil adequada do produto final. 
Como a formação do Engenheiro Civil possibilita a sua atuação em todos os segmentos atingidos pela referida norma, optou-se pela inserção de conteúdos que vão além, como todo o ramo de qualidade aplicada na construção civil. Que, entre outros conceitos, aborda a efetividade de processos e produtos, de acordo com requisitos e/ou a expectativa do cliente. Ou seja, a disciplina aqui descrita, aborda assuntos complementares em um mesmo momento, trazendo teoria e aplicabilidade aos estudantes.

Os RAs também levaram em consideração os procedimentos gerais de grandes empresas de engenharia, que têm como principal pilar, uma boa gestão de qualidade. Espera-se do estudante que ele seja capaz de desenvolver todos os procedimentos de gestão de qualidade em uma empresa de Engenharia Civil de pequeno ou médio porte.

Quadro 5 - TE e RA da disciplina de Qualidade e Desempenho das Edificações.

\begin{tabular}{|c|c|c|c|}
\hline \multicolumn{4}{|c|}{ Disciplina de Qualidade e Desempenho das Edificações (Carga Horária total de 60 horas) } \\
\hline \multicolumn{3}{|r|}{ Temas de Estudo: } & $\mathrm{CH}(\mathrm{h})$ \\
\hline TE 1 & \multicolumn{2}{|r|}{ Produtividade da mão-de-obra: Razão Unitária de Produtividade (RUP) e outros índices } & 10 \\
\hline TE 2 & \multicolumn{2}{|r|}{$\begin{array}{l}\text { Recursos humanos na construção civil: Capacitação da mão-de-obra, Formação de equipe } \\
\text { básica, Alocação dos recursos humanos. }\end{array}$} & 10 \\
\hline TE 3 & \multicolumn{2}{|c|}{$\begin{array}{l}\text { Sistema de Gestão da qualidade aplicados à construção civil: procedimentos e normas } \\
\text { técnicas. }\end{array}$} & 10 \\
\hline TE 4 & \multicolumn{2}{|r|}{ Norma de Desempenho de Edificações: requisitos, aplicações e avaliações. } & 15 \\
\hline TE 5 & \multicolumn{2}{|r|}{ Cronograma físico-financeiro e curva $\mathrm{S}$. } & 10 \\
\hline TE 6 & & Modelagem paramétrica aplicada ao gerenciamento de obras & 5 \\
\hline \multicolumn{4}{|c|}{ Resultados de Aprendizagem: } \\
\hline $\mathrm{C}$ & ECs & \multicolumn{2}{|l|}{ RAs } \\
\hline \multirow[t]{2}{*}{$\begin{array}{l}\frac{\tilde{J}}{0} \\
\overline{0} \\
\dot{0} \\
0\end{array}$} & Elaborar & \multicolumn{2}{|c|}{$\begin{array}{l}\text { RA } 1 \text { (relacionado ao TE 1): Elaborar um plano de ação para serviços de construção civil } \\
\text { considerando a índices de produtividade em um cenário proposto de forma organizada e } \\
\text { eficiente. } \\
\text { RA } 4 \text { (relacionado ao TE 4): Propor alternativas para adequação de uma obra à norma de } \\
\text { desempenho considerando dados de projeto e/ou de campo de forma criativa e assertiva. } \\
\text { RA } 5 \text { (relacionado ao TE 5): Elaborar um cronograma físico-financeiro de um } \\
\text { empreendimento proposto considerando as mídias especializadas por meio de ferramentas } \\
\text { tecnológicas de forma organizada e criativa. } \\
\text { RA } 6 \text { (relacionado ao TE 6): Descrever as etapas essenciais para modelagem paramétrica } \\
\text { de uma edificação residencial proposta considerando o gerenciamento e a qualidade da } \\
\text { construção civil de forma autônoma. }\end{array}$} \\
\hline & Monitorar & $\begin{array}{l}\text { RA } 2 \text { (relacionado ao TE 2): Compatibilizar recursos humanos consideran } \\
\text { monitoramento e retroanálise da produtividade e dos prazos por meio de ferra } \\
\text { tecnológicas de forma viável e eficiente. } \\
\text { RA } 3 \text { (relacionado ao TE 3): Criar registro padronizado para coleta de informa } \\
\text { equipamentos, serviços e gestão de resíduos considerando as normas de qualic } \\
\text { legislação vigente de forma organizada e assertiva. }\end{array}$ & $\begin{array}{l}\text { o } \\
\text { entas } \\
\text { Des de } \\
\text { de e a }\end{array}$ \\
\hline
\end{tabular}

Fonte: os autores.

\section{CONSIDERAÇÕES FINAIS}

Todo o processo de desenvolvimento de TEs e RAs, baseados na inovação curricular proposta, despertou nos professores envolvidos o interesse em um aprimoramento nas metodologias de ensino. Muitos deles têm procurado conhecer mais metodologias, principalmente as ativas, a fim de aperfeiçoar o processo de ensino de suas disciplinas. Isso, com certeza, irá resultar em uma melhora na qualificação do aluno egresso.

Uma característica peculiar da área de Construção Civil é a sua abrangência dentro do curso contribuindo com o desenvolvimento de todas as competências e de 25 dos 39 ECs. Demonstrando que esta é a maior área do curso de Engenharia Civil da UTFPR, campus Guarapuava. 
A expectativa desse grupo de professores, que vêm construindo esse PPC, é de que o egresso do curso de Engenharia Civil da UTFPR Guarapuava seja devidamente qualificado para atender todas as atribuições que o CONFEA regulamenta. Mas que, também, saiba encontrar o caminho de conhecimento para seu contínuo aperfeiçoamento, de acordo com seus próprios interesses. Por isso, entende-se a importância de forjar no estudante, a maturidade necessária para vencer os desafios que encontrará em sua trajetória, e que vão muito além do simples conhecimento técnico. O egresso deste curso tem contato com exemplos práticos de pequenas, médias e grandes empresas, indústria e fornecedores de materiais, projetistas, construtores, peritos, empreendedores, além da formação humanística e de comunicação oferecidas, que, espera-se completar a formação de um profissional melhor preparado para o mercado e seus desafios.

Por fim, destaca-se a importância de que o trabalho de reestruturação curricular seja realizado de forma coletiva e colaborativa, onde os professores das diferentes áreas contribuem com conhecimentos inerentes à sua formação específica e, também, por meio de suas experiências e vivências pretéritas. O apoio do Departamento de Educação do campus, representado pela participação da pedagoga durante o processo, também teve fundamental relevância para o aprimoramento dos temas de estudo e dos resultados de aprendizagem desenvolvidos.

\section{Agradecimentos}

Os autores agradecem a UTFPR por ter fornecido a oportunidade de aprendizado e incentivo a inovação curricular através da Oficina de Design de Cursos oferecida pelo Departamento de Educação (DEPEDUC) da Pró-reitoria de Graduação (PROGRAD).

E aos colegas de NDE e colegiado que ajudaram na construção e desenvolvimento da nova matriz curricular do curso de Engenharia Civil da UTFPR, campus Guarapuava.

\section{REFERÊNCIAS}

BRASIL, Diretrizes Curriculares Nacionais do Curso de Graduação em Engenharia, Resolução no 2/2019 do Ministério da Educação, de 24 de Abril de 2019.

LOPES, Alice Casimiro. Políticas de integração curricular. Rio de Janeiro: UERJ, 2008

SCALLON, Gérard. Avaliação da aprendizagem numa abordagem por competências. Tradução de Juliana Vermelho Martins. Curitiba: PUCPRess, 2015.

UTFPR, Projeto Pedagógico Institucional (PPI) da Universidade Tecnológica Federal do Paraná, Curitiba, 2019. 


\title{
TEACHING BY SKILLS IN THE DISCIPLINES OF THE CIVIL CONSTRUCTION AREA - CASE OF UTFPR GUARAPUAVA
}

\begin{abstract}
The changes required for Engineering undergraduate courses are motivated by scientific and technological advances and in the transformation of society's relationships and needs. The publication of the new National Curriculum Guidelines for the Undergraduate Engineering Course sought to synthesize the needs for change and boosted the process of curricular innovation in engineering courses in the country, as well as at the Federal Technological University of Paraná - UTFPR. The concept of training engineers needs to achieve professional skills and means mobilizing and integrating knowledge in realistic use situations, with a social, cultural, economic and scientific context. It was necessary to renew the perspectives in order to transform the curricular proposal with a competency-based approach that includes not only know (knowledge), but also know-how to do and know-how to be. This paper presents the strategies that were adopted in the reformulation of the curriculum with regard to the disciplines of the Civil Construction area of the Civil Engineering Course at UTFPR, Guarapuava campus. In addition to exposing the work methodology applied by the Structuring Teaching Nucleus of the course, three subjects in the area are presented as an example, where the themes of studies and learning results have peculiar or innovative characteristics.
\end{abstract}

Keywords: Curriculum Guidelines. Competency Curriculum. Civil Construction. 